\title{
Intramolecular Charge Transfer of $p$-(Dimethylamino)benzethyne: A Case of Nonfluorescent ICT State
}

\author{
Nitin Chattopadhyay, $*, \dagger,+, \S$ Carlos Serpa, ${ }^{\dagger}$ Mariette M. Pereira, ${ }^{\dagger}$ J. Seixas de Melo, ${ }^{\dagger}$ \\ Luis G. Arnaut, ${ }^{*} \dagger$ and Sebastião J. Formosinho ${ }^{\dagger}$ \\ Department of Chemistry, Coimbra University, P 3004-535, Coimbra, Portugal, and Department of Chemistry, \\ Jadavpur University, Calcutta 700 032, India
}

Received: June 21, 2001; In Final Form: August 20, 2001

\begin{abstract}
The effect of solvent polarity and external heavy atom on the steady state and time-resolved fluorescence properties of the locally excited (LE) state of $p$-(dimethylamino)benzethyne (DMABE) has been studied. It has been observed that in less polar environments, intersystem crossing (ISC) is the main nonradiative channel of deactivation for the excited species. However, at higher solvent polarity $\left(E_{\mathrm{T}}(30) \geq 44\right)$ the activation barrier dependent intramolecular charge transfer (ICT) reaction is the main nonradiative process dominating over ISC. The results reveal that DMABE undergoes an ICT reaction in polar environments to reach a nonfluorescent intramolecular charge transfer (ICT) state. The efficiency of formation of the ICT state of DMABE is compared with that of $p$-(dimethylamino)benzonitrile (DMABN). The present work settles the controversy raised by two previous works with conflicting results: a theoretical one, predicting that DMABE will undergo ICT reaction even under isolated conditions (Sobolewski, A. L.; Domcke, W. Chem. Phys. Lett. 1996, 259, 119), and an experimental one, excluding the possibility of the ICT process for the same molecular system (Zachariasse, K. A.; Grobys, M.; Tauer, E. Chem. Phys. Lett. 1997, 274, 382).
\end{abstract}

\section{Introduction}

The dual luminescence of $p$-dimethylaminobenzonitrile (DMABN) and related molecular systems in solutions, discovered in early $1960 \mathrm{~s},{ }^{3}$ has received immense attention due mainly to two aspects: (i) its model function affecting application in diverse fields, including laser dyes, isomerization of polyenes and rhodopsin, molecular switching devices, and charge separation in photochemical energy utilization, and (ii) the mechanistic aspect of the photoprocess. ${ }^{4-8}$ Although, since the discovery of this phenomenon, DMABN has been used as the prime molecular system for such studies, other new molecular systems have been synthesized and investigated. Among the several mechanisms proposed to explain this phenomenon, ${ }^{9-17}$ the most widely accepted mechanism was first put forward by Grabowski et al. ${ }^{15-17}$ According to this mechanism, electronic excitation leads to an intramolecular charge transfer from the donor $-\mathrm{N}\left(\mathrm{CH}_{3}\right)_{2}$ group to the acceptor end accompanied by the twisting of the former group to a conformer nearly perpendicular to the phenyl plane.

Although existence of locally excited (LE) and intramolecular charge transfer (ICT) states is widely recognized, controversy still persists regarding the mechanistic aspect of the ICT process. Recently a related molecular system, $p$-(dimethylamino)benzethyne (DMABE), has given birth to a new controversy regarding the occurrence of the ICT process itself. On the basis of ab initio electronic structure calculations on DMABN and DMABE, Sobolewski and Domcke have claimed that DMABE is more predisposed to form the ICT state than DMABN. ${ }^{1}$ They further predicted that DMABE should undergo an exothermic ICT reaction, even under isolated-molecule condition. On the other hand, the photostationary and time-resolved fluorescence

\footnotetext{
† Coimbra University.

$\doteqdot$ E-mail: pcnitin@yahoo.com

$\S$ Jadavpur University.
}

measurements of Zachariasse and co-workers did not reveal the dual fluorescence that should be the signature of ICT in DMABE. ${ }^{2}$ The absence of a second fluorescence led these authors to conclude that the ICT reaction does not occur in this molecular system under any condition of solvent polarity.

To resolve the controversy arising out of these two contradicting reports, ${ }^{1,2}$ in the present work, we have studied the steady state and time-resolved fluorescence of DMABE in different liquid environments. Our experimental observations lead to the conclusion that in reasonably polar environment $I C T$ reaction does occur with this molecular system. However, the molecule belongs to the group of fluorophores having nonemissive ICT state.

\section{Experimental Section}

DMABE was synthesized from $p$-(dimethylamino)benzaldehyde (DMABA) following the procedure described by Akiyama et al. ${ }^{18}$ The melting point of the product was $52-53{ }^{\circ} \mathrm{C} .{ }^{1} \mathrm{H}$ $\operatorname{NMR}\left(\mathrm{CDCl}_{3}\right): \delta 2.98\left(7 \mathrm{H}, \mathrm{s}, 2 \mathrm{CH}_{3}+\mathrm{CH}\right), 6.62(2 \mathrm{H}, \mathrm{d}, J=$ $\left.9 \mathrm{~Hz}, \mathrm{H}_{\mathrm{m}}\right), 7.37\left(2 \mathrm{H}, \mathrm{d}, J=9 \mathrm{~Hz}, \mathrm{H}_{\mathrm{o}}\right)$. Although the ${ }^{1} \mathrm{H}$ NMR spectrum agrees with that reported by Zachariasse et al., ${ }^{2}$ it differs a little from that reported in the work of Akiyama et al. ${ }^{18}$ To confirm the structure, we further characterized the compound by ${ }^{13} \mathrm{C}$ NMR. ${ }^{13} \mathrm{C}\left(\mathrm{CDCl}_{3}\right): \delta 150.33\left(\mathrm{C}-\mathrm{N}\left(\mathrm{CH}_{3}\right)_{2}\right.$, arom), $133.17\left(C_{2}\right.$, arom $), 111.63\left(C_{3}\right.$, arom $), 108.65\left(C_{1} \mathrm{CCH}\right.$, arom), $84.82(\mathrm{CCH}), 74.75(\mathrm{CCH}), 40.15\left(\mathrm{~N}\left(\mathrm{CH}_{3}\right)_{2}\right)$.

DMABE was purified by repeated recrystallization from an $n$-hexane-diethyl ether mixture (3:1). DMABN (Aldrich) was purified from vacuum sublimation followed by recrystallization from ethanol. The purity of both the compounds was checked by TLC as well as from spectroscopic properties.

Spectrophotometric grade $n$-heptane, isopentane, ethanol, and $p$-dioxane were purchased from Aldrich. The first three solvents were used as received. Dioxane was passed through activated 


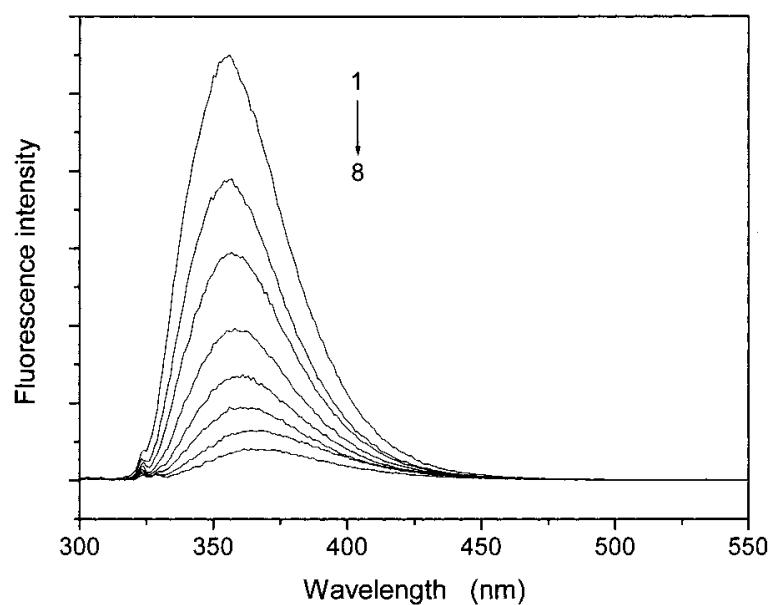

Figure 1. Fluorescence spectra of DMABE in different dioxane-water mixed solvents. The volume compositions of dioxane and water corresponding to curves 1 to 8 are 100/0, 95/5, 90/10,80/20,70/30, $60 / 40,50 / 50$ and $40 / 60$, respectively. $\lambda_{\text {excitation }}=295 \mathrm{~nm}$.

alumina and the final trace of water was removed by refluxing over sodium. The dry solvent was stored in the presence of molecular sieves. Ethyl iodide (Riedel-de Haën) was used as received. Millipore Milli-Q water was used for the preparation of the aqueous dioxane solutions.

A Shimadzu UV-2100 spectrophotometer and a Spex Fluorolog 3 spectrofluorometer were used for the absorption and fluorescence measurements, respectively. The $77 \mathrm{~K}$ phosphorescence spectra were measured in the same spectrofluorimeter with 1934D4 module for phosphorimetry. The fluorescence quantum yields (uncorrected for refractive index) of DMABE and $\mathrm{DMABN}$ in different solvents were measured against a quinine sulfate solution in $0.1 \mathrm{~N} \mathrm{H}_{2} \mathrm{SO}_{4}$ solution $(\phi=0.546)^{19}$ as well as a DMABN solution in $n$-hexane $(\phi=0.20) .{ }^{20}$ All the solutions were freshly prepared just before the experiments. For both steady-state and time-resolved measurements, the solutions were degassed by passing argon for $20 \mathrm{~min}$.

Fluorescence lifetimes were measured by the time-correlated single photon counting (TCSPC) technique with an apparatus using an IBH 5000 coaxial flashlamp (filled with $\mathrm{N}_{2}$ ) as the excitation source, a Philips XP2020Q photomultiplier, a JobinIvon H20 monochromator, and a Canberra Instruments timeto-amplitude converter and multichannel analyzer. Alternate measurements of the lamp profile and sample emission were performed. The fluorescence decays were analyzed using the method of modulating functions of Stricker, ${ }^{21}$ with automatic correction for the photomultiplier "wavelength shift". The detail of the TCSPC set up has been described elsewhere. ${ }^{22}$ The time resolution of our equipment is ca. 200 ps. This instrumental limitation restricts us to measure lifetimes of the DMABN system in solutions with $E_{\mathrm{T}}(30)>46.3$, as the measured lifetimes fall within the time resolution of our instrumentation, limiting the reliability of the measured data.

\section{Results and Discussion}

The fluorescence spectra of DMABE $\left(\right.$ ca. $\left.1 \times 10^{-5} \mathrm{M}\right)$ as a function of solvent polarity $\left(E_{\mathrm{T}}(30)\right)$ in a series of mixtures of dioxane and water is given in Figure 1. The fluorophore gives rise to a single fluorescence band with a maximum at around $355 \mathrm{~nm}$.

From a mirror relationship between the absorption and emission spectra, and from comparison with the emission spectra of similar class of molecules, this emission can safely be assigned to an emission from the locally excited (LE) state of
TABLE 1: Steady-State and Kinetic Fluorometric and Nonradiative Parameters of DMABE and DMABN in Alkane and Different Dioxane-Water Solutions

\begin{tabular}{|c|c|c|c|c|c|c|}
\hline dioxane/water & $\begin{array}{c}E_{\mathrm{T}}(30)^{a} \\
(\mathrm{kcal} / \mathrm{mol})\end{array}$ & $\phi_{\mathrm{F}}(\mathrm{LE})$ & $\begin{array}{c}\tau_{\mathrm{LE}} \\
(\mathrm{ns})\end{array}$ & $\begin{array}{c}10^{-7} k_{\mathrm{F}} \\
\left(\mathrm{s}^{-1}\right)\end{array}$ & $\begin{array}{c}10^{-8} k_{\mathrm{NR}} \\
\left(\mathrm{s}^{-1}\right)\end{array}$ & $\ln \left(k_{\mathrm{NR}}\right)$ \\
\hline \multicolumn{7}{|c|}{ DMABE } \\
\hline$n$-hexane & 31.0 & $0.28^{b}$ & $5.05^{b}$ & 5.5 & 1.4 & 18.77 \\
\hline$n$-heptane & 31.1 & 0.260 & 4.70 & 5.5 & 1.5 & 18.83 \\
\hline $100 / 0$ & 36.6 & 0.217 & 3.70 & 5.8 & 2.1 & 19.16 \\
\hline $95 / 5$ & 43.8 & 0.152 & 2.67 & 5.7 & 3.2 & 19.58 \\
\hline $90 / 10$ & 46.3 & 0.117 & 2.10 & 5.6 & 4.2 & 19.86 \\
\hline $80 / 20$ & 49.2 & 0.081 & 1.24 & 6.5 & 7.4 & 20.42 \\
\hline $70 / 30$ & 50.8 & 0.057 & 0.92 & 6.2 & 10.2 & 20.74 \\
\hline $60 / 40$ & 52.1 & 0.040 & 0.65 & 6.2 & 14.8 & 21.11 \\
\hline $50 / 50$ & 53.4 & 0.031 & 0.46 & 6.7 & 21.1 & 21.47 \\
\hline $40 / 60$ & 55.8 & 0.019 & 0.31 & 6.1 & 31.6 & 21.87 \\
\hline \multicolumn{7}{|c|}{ DMABN } \\
\hline$n$-hexane & 31.0 & $0.20^{c}$ & $2.9^{c}$ & 6.9 & 2.76 & 19.4 \\
\hline$n$-heptane & 31.1 & 0.19 & 3.4 & 5.6 & 2.34 & 19.27 \\
\hline $100 / 0$ & 36.6 & 0.039 & 3.68 & 1.1 & 2.60 & 19.38 \\
\hline $95 / 5$ & 43.8 & 0.006 & 0.32 & 1.9 & 31.06 & 21.86 \\
\hline $90 / 10$ & 46.3 & 0.0023 & 0.20 & 1.2 & 38.32 & 22.07 \\
\hline $80 / 20$ & 49.2 & 0.0011 & $d$ & & & \\
\hline $70 / 30$ & 50.8 & 0.00068 & $d$ & & & \\
\hline $60 / 40$ & 52.1 & 0.00063 & $d$ & & & \\
\hline $50 / 50$ & 53.4 & 0.00060 & $d$ & & & \\
\hline $40 / 60$ & 55.8 & 0.00050 & $d$ & & & \\
\hline
\end{tabular}

${ }^{a}$ From refs 23 and 24. ${ }^{b}$ From ref 2. ${ }^{c}$ From ref 25. ${ }^{d}$ Lifetimes could not be measured as they were within the time resolution of our instrument.

the fluorophore. With an increase in the solvent polarity the emission maximum shifts a little toward longer wavelengths in more polar environment. The most interesting observation from Figure 1 is, however, an enormous decrease in the fluorescence quantum yield with an increase in the solvent polarity. Unlike DMABE, DMABN yields two bands in its fluorescence spectra. As suggested by the small solvent-induced Stokes shift, the emission around $350 \mathrm{~nm}$ corresponds to the LE state. The other broad band situated around $425 \mathrm{~nm}$ in dioxane solution is assigned to the ICT and shows a dramatic red shift, characteristic of a charge-transfer band, in more polar environments. ${ }^{3,4}$ In the case of DMABN, spectral decomposition leading to the fluorescence yields of each state was performed by Gaussian fittings to the LE and CT bands. The fluorescence quantum yields $\left(\phi_{\mathrm{F}}\right)$ of the LE states of DMABE and DMABN in different environments are presented in Table 1.

The fluorescence decay measurements with DMABE reveal that these are always single exponential. Using the flash lamp excitation, we did not find any degradation of the compound in any of the environments. This is consistent with the previous literature report. ${ }^{2}$ For DMABN, however, the decays were biexponential, giving a signature of the reversibility of the ICT process (LE $\leftrightarrow$ ICT). The major component gives the lifetime of the LE state. The fluorescence lifetimes of the LE states $\left(\tau_{\mathrm{LE}}\right)$ of DMABE and DMABN in different environments are also presented in Table 1.

Figure 2 represents some of the typical fluorescence decays of DMABE in different dioxane-water mixtures. Figure 2 and Table 1 indicate that with an increase in the solvent polarity $\left(E_{\mathrm{T}}(30)\right)$ the fluorescence lifetime decreases continuously over the $E_{\mathrm{T}}(30)$ range used in the present study.

The fluorescence decay rate in each solvent, $k_{\mathrm{F}}=\phi_{\mathrm{F}} / \tau_{\mathrm{LE}}$, where $\tau_{\mathrm{LE}}=1 /\left(k_{\mathrm{F}}+k_{\mathrm{IC}}+k_{\mathrm{ISC}}+k_{\mathrm{ICT}}\right)$, and the nonradiative rate, $k_{\mathrm{NR}}=k_{\mathrm{IC}}+k_{\mathrm{ISC}}+k_{\mathrm{ICT}}$, are presented in the same table. The large decrease in $\phi_{\mathrm{F}}$ of DMABE corresponds to a remarkable increase in the nonradiative rate $\left(k_{\mathrm{NR}}\right)$, from $2.1 \times 10^{8} \mathrm{~s}^{-1}$ 


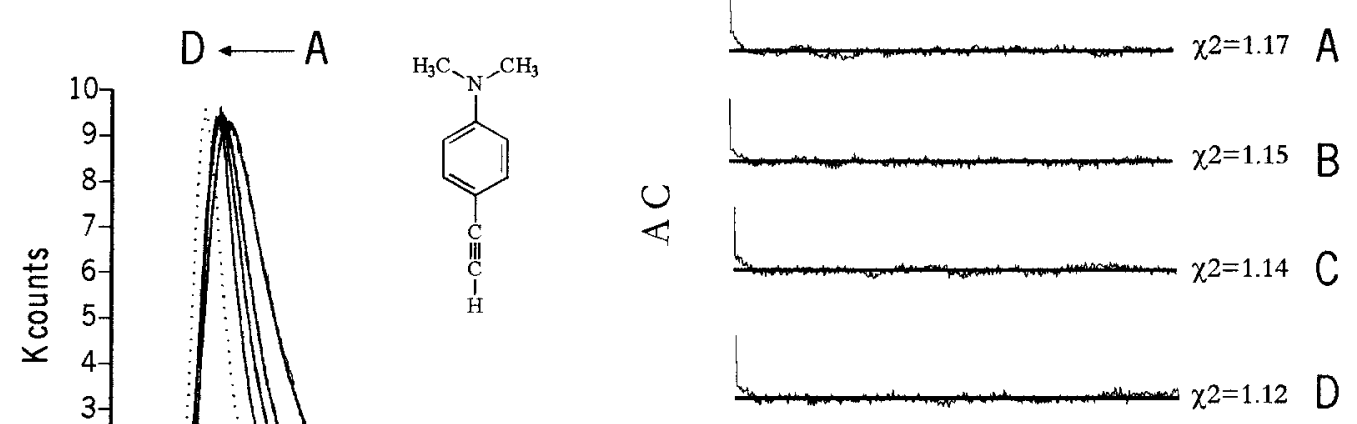

$96.6 \mathrm{ps} / \mathrm{ch}$

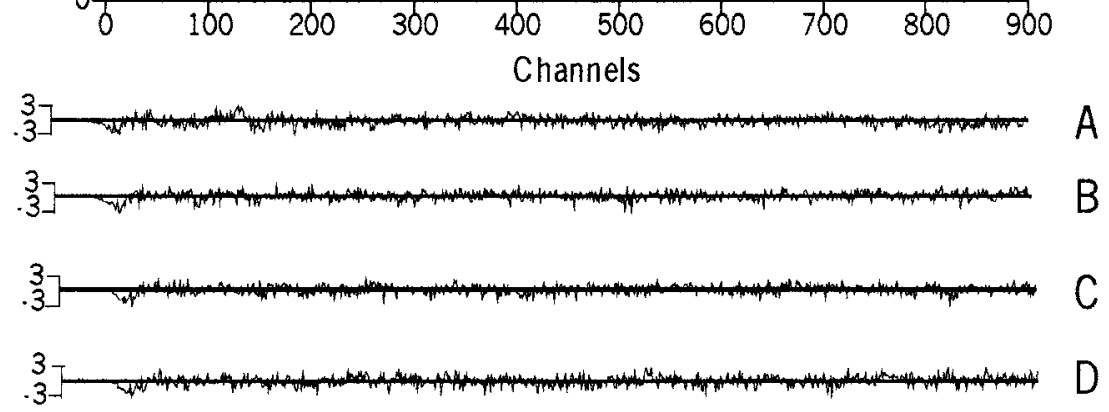

\section{Weighted Residuals}

Figure 2. Some typical fluorescence decays of DMABE in dioxane-water environments: (A-D) 100/0, 90/10, 80/20, and 50/50 mixtures of dioxane and water $(\mathrm{v} / \mathrm{v})$, respectively. The dotted line gives the pulse instrumental response. $\lambda_{\mathrm{exc}}=316 \mathrm{~nm}$, and $\lambda_{\mathrm{em}}=360 \mathrm{~nm}$. $\chi^{2}$, autocorrelation function (AC), and weigthed residuals (WR) are shown to judge the quality of the fit.

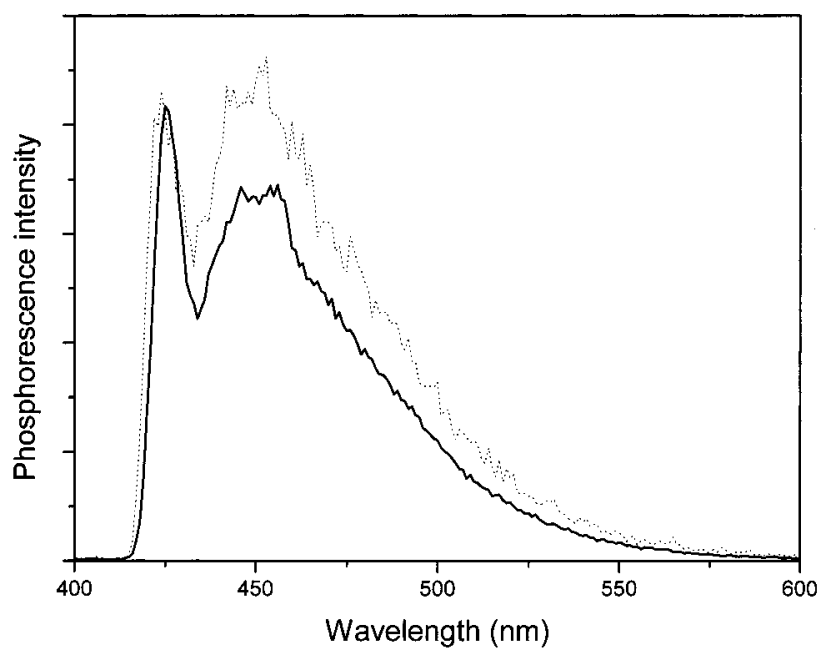

Figure 3. $77 \mathrm{~K}$ phosphorescence spectra of DMABE in isopentane $(\cdots)$ and ethanol ( - ) glass. $\lambda_{\text {excitation }}=295 \mathrm{~nm}$.

in pure dioxane to $31.6 \times 10^{8} \mathrm{~s}^{-1}$ in $40 \%$ dioxane-water (ref. to Table 1 ), while the radiative rate is not affected significantly with this change in the solvent polarity. ${ }^{26-31}$

The strong polarity dependence of the nonradiative rate cannot be due to internal conversion (IC) or intersystem crossing (ISC), which depend on the energy gap between the singlet excited state and lower triplets or ground states, ${ }^{32}$ as those rates under the present experimental conditions can change at most by a few percent. We have determined the triplet state energies of DMABE in isopentane and ethanol glass matrices from the 77 $\mathrm{K}$ phosphorescence spectra (Figure 3). The energy values determined are 282 and $281 \mathrm{~kJ} \mathrm{~mol}^{-1}$, respectively in the two environments. The proximity of these energy values reveals that the triplet state energy does not depend appreciably on the

\section{SCHEME 1}

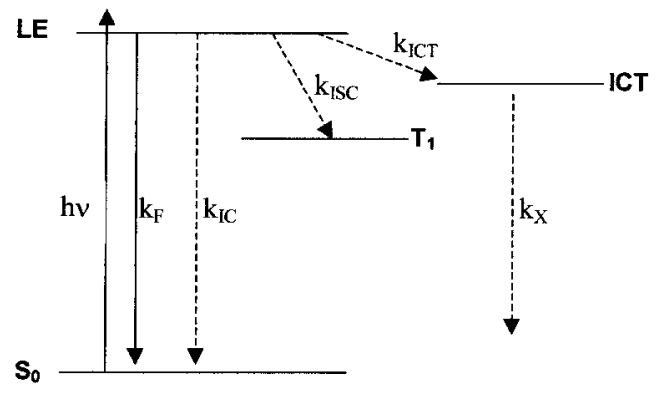

solvent polarity. A specific interaction or reaction between the excited fluorophore and solvent is also ruled out from the observation of similar polarity dependence in other solvents. ${ }^{2}$ The only process known in the literature that exhibits such a strong polarity dependence is the twisted intramolecular charge transfer (TICT) process. ${ }^{6,7,33,34}$ Since the structure of DMABE satisfies the criterion for the TICT process to occur, with an electron donor (dimethylamino) and an aromatic acceptor moiety joined by a flexible bond (single bond), we propose that polarity dependent ICT is the main nonradiative process for the LE state in highly polar environments. DMABE does not give rise to dual fluorescence, which led Zachariasse et al. to conclude that the ICT reaction does not occur with this molecular system. ${ }^{2}$ However, for a large number of systems related to DMABE, ICT to form a nonemissive ICT state has been suggested. ${ }^{6,28-31,33-37}$

We can explain the polarity dependence of the nonradiative deactivation of DMABE, with Scheme 1, by analogy with the mechanism proposed by Grabowski et al.. ${ }^{15,16}$ In Scheme $1, \mathrm{~S}_{0}$, LE, $\mathrm{T}_{1}$ and ICT are the ground state, the locally excited singlet state, the lowest triplet state, and the intramolecular charge 
transfer state, respectively. The fluorescence rate of the LE state is represented by $k_{\mathrm{F}}$. The different nonradiative rates of the LE state are $k_{\mathrm{IC}}$ (internal conversion), $k_{\mathrm{ISC}}$ (intersystem crossing), and $k_{\mathrm{ICT}}$ (intramolecular charge transfer). $k_{\mathrm{X}}$ represents the total decay rate of the ICT state.

The fluorophore in the ground state $\left(\mathrm{S}_{0}\right)$ is first photoexcited to the locally excited (LE) state with a molecular geometry quite similar to that of the ground state. This LE state gives rise to the LE emission maintaining a mirror relationship with the absorption spectrum. In the ICT process, the molecule undergoes a transition from the LE state to the ICT state with a given activation barrier. Since the ICT state is considerably more polar that the LE state, ${ }^{1,2}$ this activation barrier for the ICT process decreases with an increase in the medium polarity. ${ }^{6,7}$ The ICT state is nonemissive, presumably because of an efficient intersystem crossing to the triplet. ${ }^{24,32}$ In principle, Scheme 1 should account for the reversibility of the ICT process; that is, it should consider the formation of the LE state from the ICT state. However, the absence of fluorescence from the ICT state suggests that there is a very effective nonradiative decay channel available for the ICT state, and that dominates over the ICT $\rightarrow$ LE channel in the DMABE system.

Eisenthal and co-workers showed that the polarity dependence of ICT in DMABN could be explained by assuming that the activation energy for this process decreased linearly with an increase in solvent polarity. When the $E_{\mathrm{T}}(30)$ scale is employed, ${ }^{33,34}$ the relevant equation takes the following form

$$
E_{\mathrm{A}}=E_{\mathrm{A}}{ }^{0}-\alpha\left[E_{\mathrm{T}}(30)-30\right]
$$

where $E_{\mathrm{A}}{ }^{0}$ is the barrier height in a nonpolar solvent having $E_{\mathrm{T}}(30)$ of $30 \mathrm{kcal} / \mathrm{mol}$ and $\alpha$ is a dimensionless parameter that determines how strongly $E_{\mathrm{A}}$ varies with solvent polarity. Following this formulation, the dependence of $k_{\mathrm{ICT}}$ on $E_{\mathrm{T}}(30)$ can be expressed in terms of a reference solvent with $E_{\mathrm{T}}(30)=$ $30 \mathrm{kcal} / \mathrm{mol}, k^{0}$ ICT, and an exponential dependence on the difference of $E_{\mathrm{T}}(30)$ values,

$$
k_{\mathrm{ICT}}=k_{\mathrm{ICT}}^{0} \exp \left\{\alpha\left[E_{\mathrm{T}}(30)-30\right] / R T\right\}
$$

The solvent dependence of $k_{\mathrm{NR}}$ can be calculated using eq 2 assuming that $\left(k_{\mathrm{IC}}+k_{\mathrm{ISC}}\right)$ is independent of the solvent polarity. The values of $\left(k_{\mathrm{IC}}+k_{\mathrm{ISC}}\right)$ for both DMABE and DMABN can be estimated from the data in $n$-hexane ${ }^{2,25}$ and $n$-heptane, where $k_{\mathrm{ICT}} \ll\left(k_{\mathrm{IC}}+k_{\mathrm{ISC}}\right)$. We estimate a value of $\left(k_{\mathrm{IC}}+k_{\mathrm{ISC}}\right)=1.5$ $\times 10^{8} \mathrm{~s}^{-1}$ for DMABE and $2.5 \times 10^{8} \mathrm{~s}^{-1}$ for DMABN. Eisenthal et al. estimated $\left(k_{\mathrm{IC}}+k_{\mathrm{ISC}}\right)=3 \times 10^{8} \mathrm{~s}^{-1}$ for DMABN. ${ }^{33}$ Equation 2 involves only two adjustable parameters, $k_{\text {ICT }}^{0}$ and $\alpha$. They can be obtained from the fit of the solvent dependence of $k_{\mathrm{NR}}$. Figure 4 compares the experimental and calculated nonradiative rates in semilogarithmic form as a function of $E_{\mathrm{T}}(30)$. We have used $k^{0} \mathrm{ICT}=1 \times 10^{7} \mathrm{~s}^{-1}$ for both DMABE and DMABN. This is in very good agreement with the value obtained by Eisenthal et al. for DMABN, $2 \times 10^{7}$ $\mathrm{s}^{-1}$. Chang and Cheung applied a similar kinetic mechanism to explain the solvent polarity dependence of nonradiative rates of Rhodamine $\mathrm{B}$ and obtained $k^{0} \mathrm{ICT}$ ( $k_{\text {cor }}$ in their nomenclature) equal to $1.6 \times 10^{7} \mathrm{~s}^{-1} \cdot{ }^{28,29}$ Using the same method, we calculate $k_{\text {ICT }}^{0}=1.13 \times 10^{7} \mathrm{~s}^{-1}$ with a standard deviation of about $6 \%$ of the mean (Table 2). According to Chang and Cheung, this is evidence of the fact that all the essential factors have been taken into account in the calculation of the nonradiative rates. The solvent dependence of DMABN is much larger than that of DMABE. This is manifested by $\alpha=0.23$ and 0.13 for DMABN

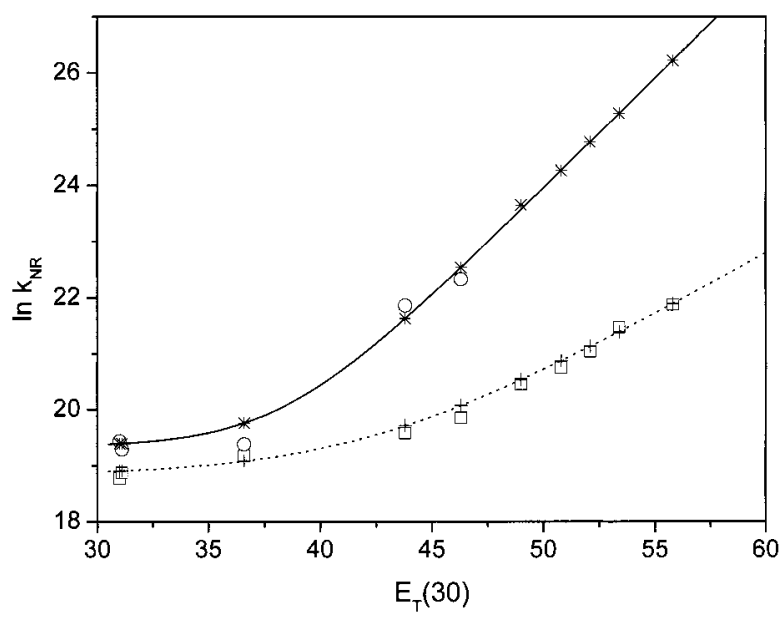

Figure 4. Semilogarithmic plot of the nonradiative rate of DMABE and DMABN vs solvent polarity expressed as $E_{\mathrm{T}}(30)$ in $n$-hexane, $n$-heptane, and a series of dioxane-water mixed solvents. Open squares and circles represent the experimental data points for DMABE and DMABN, respectively. Pluses and stars are the calculated points at the experimental $E_{\mathrm{T}}(30)$ 's. The broken and the solid lines give the calculated trends for the two molecular systems, respectively, in the experimental $E_{\mathrm{T}}(30)$ range. The fluorescence lifetimes of DMABN in solutions with $E_{\mathrm{T}}(30)>46.3$ are within the time resolution of our instrumentation (ca. $200 \mathrm{ps}$ ), restricting us to get data points beyond this polarity.

TABLE 2: Solvent Polarity Corrected Nonradiative Rate $\left(k_{\text {cor }}\right)$ of DMABE in Different Dioxane-Water Solutions (in the Linear Range of Figure 4)

\begin{tabular}{ccc}
\hline dioxane/water $(\mathrm{v} / \mathrm{v})$ & $\begin{array}{c}E_{\mathrm{T}}(30) \\
(\mathrm{kcal} / \mathrm{mol})\end{array}$ & $\begin{array}{c}10^{-7} k_{\text {cor }} \\
\left(\mathrm{s}^{-1}\right)\end{array}$ \\
\hline $90 / 10$ & 46.3 & 1.17 \\
$80 / 20$ & 49.2 & 1.09 \\
$70 / 30$ & 50.8 & 1.07 \\
$60 / 40$ & 52.1 & 1.16 \\
$50 / 50$ & 53.4 & 1.20 \\
$40 / 60$ & 55.8 & 1.10
\end{tabular}

and DMABE, respectively. Eisenthal et al. showed that, for DMABN, the ICT process is reversible in the range $33<E_{\mathrm{T}^{-}}$ $(30)<42$, essentially because the energies of the LE and ICT states are quite close. In the present study, only the results obtained in pure dioxane fall in this range and this does not obscure the trend exhibited by the data. The larger value of $\alpha$ for DMABN reveals that this molecule forms ICT states even in less polar solvents than DMABE. For DMABN, ICT becomes the main nonradiative deactivation channel for the LE state at $E_{\mathrm{T}}(30) \geq 40$. The value of $\alpha$ obtained for DMABE is, however, not negligible and ICT becomes the dominant deactivation channel of the LE state at $E_{\mathrm{T}}(30) \geq 44$.

As an obvious outcome of our general approach, Figure 4 reveals that at higher solvent polarity (leading to $k_{\mathrm{ICT}} \gg\left(k_{\mathrm{IC}}+\right.$ $k_{\mathrm{ISC}}$ ) and hence $k_{\mathrm{NR}} \approx k_{\mathrm{ICT}}$ ) the semilogarithmic plot follows a linear trend. Such linearity, in the high polar environments, has been established for a number of similar fluorophore systems having a nonemissive ICT state. ${ }^{26-31}$

The $\phi_{\mathrm{F}}$ values calculated with these two adjustable parameters are also in very good agreement with the experimental ones, when the experimental $k_{\mathrm{F}}$ values are employed in the calculations (Figure 5). The apparently different behavior of DMABN and DMABE is explained by the same argument.

Both Figures 4 and 5 suggest that DMABN is more predisposed than DMABE to undergo ICT reaction. Since the two molecular systems are quite alike structurally, a comparison of the electron affinities or redox potentials of these two systems 


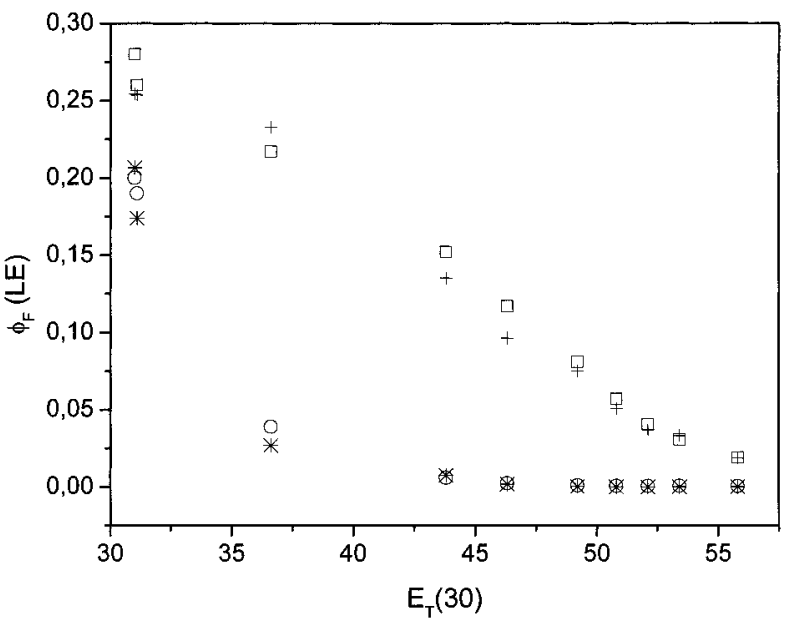

Figure 5. Calculated and experimental dependence of $\phi_{\mathrm{F}}$ on the solvent polarity parameter $E_{\mathrm{T}}(30)$ in $n$-hexane, $n$-heptane, and dioxane-water mixtures. Open squares and circles represent the experimental data points for DMABE and DMABN, respectively. Pluses and stars are the calculated points. The fluorescence quantum yields were calculated with $k_{\mathrm{ICT}}^{0}=1 \times 10^{7} \mathrm{~s}^{-1}$ and $\alpha=0.23$ or 0.13 for DMABN or DMABE, respectively; $k_{\mathrm{F}}$ and $\left(k_{\mathrm{IC}}+k_{\mathrm{ISC}}\right)$ were estimated from the experimental data, as described in the text.

with $\mathrm{N}, \mathrm{N}$-dimethylaniline would probably give a directive of their relative efficiency to undergo ICT reaction. In the case of unavailability of the above data, one can also deal with the system qualitatively using the Hammett substituent constant, $\sigma$, of the groups $-\mathrm{CN}$ and $-\mathrm{CCH}$, since with many systems it has been established that the redox potential has a linear relation with $\sigma .^{38}$ The $-\mathrm{CN}$ and $-\mathrm{CCH}$ groups have $\sigma_{\mathrm{p}}$ values of +0.71 and +0.23 , respectively, for the para substitution. ${ }^{39}$ According to the definition of the $\sigma$ parameter, electron withdrawing substituents have more positive $\sigma$ values. Thus, $-\mathrm{CN}$ group has a greater affinity toward electron acceptance compared to the other group. This suggests that DMABN should be more predisposed to form the ICT state compared to DMABE, corroborating our experimental observation.

The general scheme along with Figure 4 suggests that at higher solvent polarity, ICT is the principal nonradiative process and, in less polar environments, IC and ISC are the dominating nonradiative deactivation channels. For DMABN and similar systems, it is known that in less polar media, ISC is the dominating term among these two ( $\phi_{\text {ISC }}$ is much greater than $\phi_{\text {IC })}{ }^{40}$ The intersystem crossing efficiency can be enhanced by increasing the spin-orbit coupling, e.g., via an external heavy atom effect. ${ }^{41}$ Thus, to justify the validity of the model we have further studied the effect of ethyl iodide (EtI) on the nonradiative deactivation of DMABE following the fluorescence quenching of the fluorophore and measuring the lifetime of the probe at different ethyl iodide concentrations in less polar $\left(E_{\mathrm{T}}(30)=\right.$ 36.6) and more polar $\left(E_{\mathrm{T}}(30)=52.1\right)$ environments. Table 3 records the lifetimes of the LE state of DMABE in the presence of different concentrations of ethyl iodide in both the environments. It is clear from the table that while ethyl iodide reduces the fluorescence lifetime of the probe appreciably in less polar solution, it does not have any influence in the other medium.

Figure 6 presents the relative change of $k_{\mathrm{NR}}$ of DMABE in the aforesaid two environments. The figure establishes that in the less polar environment ISC has the dominating role in the nonradiative deactivation. However, in the highly polar environment there is hardly any effect of external heavy atom on $k_{\mathrm{NR}}$, corroborating the proposal that at this solvent polarity ICT is the principal nonradiative pathway.

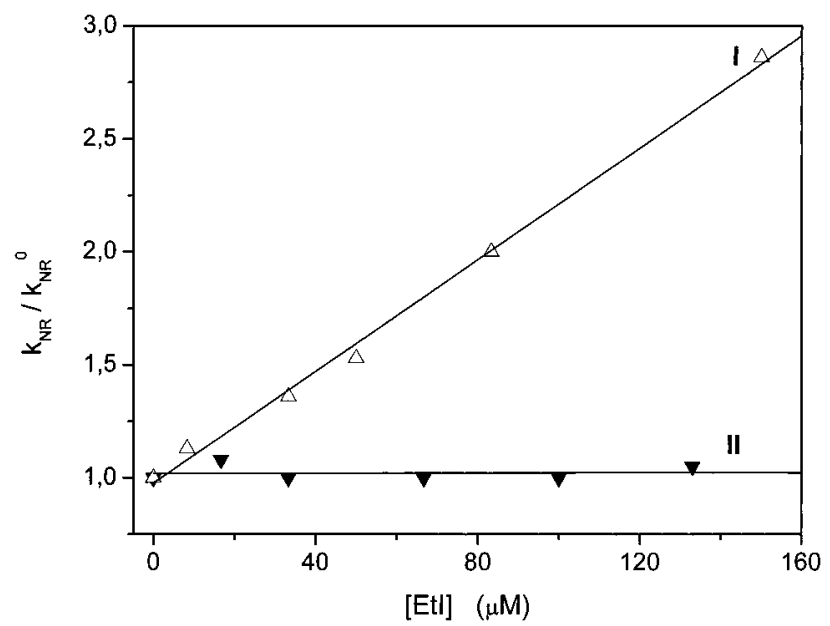

Figure 6. Plot of $k_{\mathrm{NR}} / k_{\mathrm{NR}}^{0}$ vs concentration of ethyl iodide for DMABE: (I) $E_{\mathrm{T}}(30)=36.6$; (II) $E_{\mathrm{T}}(30)=52.1$. $k_{\mathrm{NR}}{ }^{0}$ refers to the nonradiative rate in the absence of ethyl iodide.

TABLE 3: Fluorescence Lifetimes of the LE State of DMABE in the Presence of Different Concentrations of Ethyl Iodide in Two Different Liquid Environments

\begin{tabular}{ccccc}
\hline $\begin{array}{c}\text { environment } \\
\text { (dioxane/water) }\end{array}$ & $\begin{array}{c}E_{\mathrm{T}}(30) \\
(\mathrm{kcal} / \mathrm{mol})\end{array}$ & $\begin{array}{c}\text { concn of ethyl } \\
\text { iodide }(\mu \mathrm{M})\end{array}$ & $\begin{array}{c}\tau_{\mathrm{LE}} \\
(\mathrm{ns})\end{array}$ & reduced $\chi^{2}$ \\
\hline $100 / 0$ & 36.6 & 0 & 3.70 & 1.17 \\
& & 8.3 & 3.30 & 1.20 \\
& & 33.3 & 2.74 & 1.18 \\
& & 50.0 & 2.43 & 1.15 \\
$60 / 40$ & & 83.3 & 1.86 & 1.27 \\
& 52.1 & 150.0 & 1.30 & 1.28 \\
& & 0 & 0.65 & 1.02 \\
& & 16.7 & 0.60 & 1.10 \\
& & 33.3 & 0.65 & 1.02 \\
& & 66.7 & 0.65 & 1.03 \\
& & 100.0 & 0.65 & 1.03 \\
& & 133.3 & 0.62 & 1.03
\end{tabular}

\section{Conclusion}

The work reveals that the rather strong polarity dependence of the fluorescence properties of DMABE arises from the polarity dependent TICT process. Thus, the ICT process does occur with the fluorophore in polar environments. However, the ICT state is nonemissive. The work further establishes that the formation of the ICT state is less efficient for DMABE compared to DMABN, ruling out the theoretical proposition that the probe is a better candidate than DMABN for the formation of the ICT state.

Acknowledgment. Financial support from PRAXIS/PCEX/ QUI/0108/96 (European Union) and Fundação para a Ciência e a Tecnologia (grant BD/18362/98) is gratefully acknowledged. We thank Professor H. D. Burrows for helpful discussions.

\section{References and Notes}

(1) Sobolewski, A. L.; Domcke, W. Chem. Phys. Lett. 1996, 259, 119.

(2) Zachariasse, K. A.; Grobys, M.; Tauer, E. Chem. Phys. Lett. 1997, $274,382$.

(3) Lippert, E.; Lüder, W.; Boos, H. In Advances in Molecular Spectroscopy; Mangini, A., Ed.; Pergamon Press: Oxford, U.K., 1962; p 443.

(4) Lippert, E.; Rettig, W.; Bonacic-Koutecký V.; Heisel, F.; Miehe, J. A. Adv. Chem. Phys. 1987, 68, 1 .

(5) Rettig, W. Angew. Chem., Int. Ed. Engl. 1986, 25, 971.

(6) Bhattacharyya, K.; Chowdhury, M. Chem. Rev. 1993, 93, 507.

(7) Kundu, S.; Bera, S. C.; Chattopadhyay, N. Ind. J. Chem. Sect. A 1998, 37, 102.

(8) Chattopadhyay, N.; Serpa, C.; Arnaut, L. G.; Formosinho, S. J. Helv. Chim. Acta, in press. 
(9) Leinhos, U.; Kühnle, W.; Zachariasse, K. A. J. Phys. Chem. 1991, 95, 2013.

(10) Schuddeboom, W.; Jonker, S. A.; Warman, J. M.; Leinhos, U.; Kühnle, W.; Zachariasse, K. A. J. Phys. Chem. 1992, 96, 10809.

(11) Il'ichev, Y. V.; Kühnle, W.; Zachariasse, K. A. J. Phys. Chem. A 1998, 102, 5670 .

(12) Visser, R. J.; Weisenborn, P. C. M.; Van Kan P. J. M.; Huizer, B.

H.; Varma, C. A. G. O.; Warman, J. M.; De Hass, M. P. J. Chem. Soc., Faraday Trans. 2 1985, 81, 689.

(13) Weisenborn, P. C. M.; Varma, C. A. G. O.; De Hass, M. P.; Warman, J. M. Chem. Phys. Lett. 1986, 129, 562.

(14) Wang, Y.; McAuliffe, M.; Novak, F.; Eisenthal, K. B. J. Phys. Chem. 1981, 85, 3736

(15) Rotkiewicz, K.; Grellmann, K. H.; Grabowski, Z. R. Chem. Phys. Lett. 1973, 19, 315.

(16) Rotkiewicz, K.; Grellmann, K. H.; Grabowski, Z. R. Chem. Phys. Lett. 1973, 21, 212

(17) Karpiuk, J.; Grabowski, Z. R.; De Schryver, F. C. Proc. Ind. Acad. Sci. 1992, 104, 133

(18) Akiyama, S.; Nakatsuji, S.; Yoshida, K.; Nakashima, K.; Hagiwara,

T.; Tsuruta, H.; Yoshida, T. Bull. Chem. Soc. Jpn. 1983, 56, 361.

(19) Demas, J. N.; Crosby, G. A. J. Phys. Chem. 1971, 75, 991

(20) Rotkiewicz, K.; Köhler, G. J. Lumines. 1987, 37, 219.

(21) Stricker, G.; Subramaniam, V.; Seidel, C. A. M.; Volkmer, A. J. Phys. Chem. B 1999, 103, 8612.

(22) Seixas de Melo, J.; Fernandes, P. F. J. Mol. Struct. 2001, 565/566,

(23) Reichardt, C. Chem. Rev. 1994, 94, 2319.

(24) Sarkar, N.; Das K.; Nath, D.; Bhattacharyya, K. Langmuir 1994 10,326
(25) Murov, S. L.; Carmichael, I.; Hug G. L. In Handbook of Photochemistry, 2nd ed.; Marcel Dekker: New York, 1993; p 15.

(26) Kosower, E. M.; Dodiuk, H. J. Am. Chem. Soc. 1978, 100, 4173.

(27) Kosower, E. M.; Dodiuk, H.; Kanety, H. J. Am. Chem. Soc., 1978, $100,4179$.

(28) Chang, T.-L.; Cheung, H. C. Chem. Phys. Lett. 1990, 173, 343.

(29) Chang, T.-L.; Cheung, H. C. J. Phys. Chem. 1992, 96, 4874.

(30) Chang, T.-L.; Borst, H. L. J. Chem. Phys. 1990, 93, 4724.

(31) Das K.; Sarkar, N.; Nath, D.; Bhattacharyya, K. Spectrochim. Acta 1992, 48A, 1701

(32) Avouris, P.; Gelbert, W. M.; El-Sayed, M. A. Chem. Rev. 1977, 77,597

(33) Hicks, J.; Vandersall, M.; Babarogic, Z.; Eisenthal, K. B. Chem. Phys. Lett. 1995, 116, 18

(34) Hicks, J. M.; Vandersall, M. T.; Sitzmann, E. V.; Eisenthal, K. B. Chem. Phys. Lett. 1997, 135, 413.

(35) Simon, J. D.; Su S. G. J. Phys. Chem. 1990, 94, 3656.

(36) Casey, K. G.; Quitevis, E. L. J. Phys. Chem. 1988, 92, 6590.

(37) Nag A.; Bhattacharyya, K. Chem. Phys. Lett. 1990, 169, 12.

(38) Zuman, P. The Elucidation of Organic Electrode Processes; Academic Press: New York, 1969; p 119.

(39) Isaacs, N. Physical Organic Chemistry, 2nd. ed.; Longman: London, 1998; pp 152-153.

(40) Chattopadhyay, N.; Rommens, J.; Van der Auweraer, M.; De Schryver, F. C. Chem. Phys. Lett. 1997, 264, 265.

(41) Becker, R. S. Theory and Interpretation of Fluorescence and Phosphorescence; Wiley-Interscience: New York, 1969; Chapter 11. 